\title{
Editorial: Introductory remarks by Alan Watson
}

\author{
A.A. Watson ${ }^{\mathrm{a}}$ \\ Received: 30 July 2012 \\ Published online: 29 August 2012 - (C) Società Italiana di Fisica / Springer-Verlag 2012
}

While much progress has been made in a century of research in understanding cosmic rays with low to moderated energies, those with extremely high energies remain mysterious. The Pierre Auger Observatory was designed to measure the properties of cosmic rays with energies above $3 \times 10^{18} \mathrm{eV}$ with unprecedented precision and is doing this extremely successfully. More details of the Observatory are given in the first article in this issue by Lawrence Wiencke. The Observatory was conceived by Jim Cronin (University of Chicago) and myself in 1991 and our ideas led to the construction of the largest cosmic-ray detector ever built. It is based near the town of Malargüe in Mendoza Province, Argentina, and covers $3000 \mathrm{~km}^{2}$, an area about 30 times that of Paris. The instrument contains over 1600 waterCherenkov detectors spread uniformly across the area and the volume of air above the detectors is monitored by a battery of optical-type telescopes that detects the faint fluorescence radiation emitted from excited molecular-nitrogen as the cascades of particles generated by the high-energy cosmic rays traverse the atmosphere. Monitoring of the clarity of the atmosphere, the aerosol content and the cloud cover, plus the atmospheric temperature, pressure and humidity as a function of altitude, are key activities necessary to extract the cosmic-ray science and these monitoring data attracted early interest from atmospheric scientists.

By the mid-1990s a group of physicists from 12 countries, with experience in cosmic-ray physics and particle physics, had come together to attempt to raise the support needed $(\sim \$ 50 \mathrm{M}$ in capital) to fund the project. About this time Jim Cronin and I began to discuss very informally, and largely only between ourselves, the possibility that the facilities that we envisaged at the Observatory might, when completed, be made available to scientists from other disciplines to use. We had nothing very specific in mind though at the Haverah Park air-shower array in the UK, where I had worked for many years, we had made our infrastructure available to seismologists and atmospheric scientists. One thing that both Jim Cronin and I were quite adamant about was that no "extra-curricular" activities would be encouraged until the cosmic-ray instrument was fully operational which in fact did not happen until the last water-Cherenkov detector was installed in June 2008. Also we had little idea as to how we should advertise the potential of the site to scientists from other disciplines and indeed this proved to be a relatively difficult task.

In 2005 Giorgio Matthiae (INFN Roma II, then the co-spokesperson of the Auger Observatory) and Fernando Liello (Trieste) identified an opportunity within the European Union $6^{\text {th }}$ Framework Programme to improve considerably the data link between the Auger site in Malargüe and the rest of the world. By this time the data collected nightly at the fluorescence detectors and from the associated atmospheric monitoring devices were too large to be transmitted over the data-link then available and much data had to be hand-carried from the site. To land the EU grant we had to promote the opportunities that would be available to scientists from other disciplines under the umbrella title for the project of AugerAccess. Indeed the bid to the EU contained two key letters of support from Professor Guido Visconti (L'Aquila, Italy) and Professor Mike Pilling (University of Leeds, UK). Both of these distinguished atmospheric scientists were aware at this time of the richness of the atmospheric database that the Observatory had to offer and saw possibilities for its exploitation.

When the EU grant was awarded a condition of it was that we had a public web site (http: //www . augeraccess . net) and hosted a two-day meeting to promote the interdisciplinary-science possibilities. The papers presented in this Focus Point comprise a large fraction of the reports made at the meeting held at the Centre for Mathematical Science, University of Cambridge, on 18 and 19 April 2011 to discuss Interdisciplinary Science at the Pierre Auger Observatory (IS@AO). A diverse range of topics are covered including how the atmospheric conditions are monitored with LIDARs (V. Rizi et al.) and described with models (B. Keilhauer et al.). The papers also include interesting

\footnotetext{
a e-mail: a.a.watson@leeds.ac.uk
} 
measurements about cloud identification comparing satellite data with ground observations (J. Chirinos et al.) and the aerosol content of the atmosphere (K. Louedec et al.). The latter work has led to the French group agreeing a protocol with the Auger Collaboration to study aerosols at the site, linking these studies to their on-going work at the Kerguelen Islands with the aim of evaluating models of atmospheric circulation. The Fluorescence Detectors can record the time evolution of interesting atmospheric transients, for example elves that are created in the ionosphere above thunderstorms hundreds of $\mathrm{km}$ away (R. Mussa et al.). Air showers have been proposed as possible trigger mechanism for electrical discharge and W.C. Brown et al. discuss a lightning detection system that will measure the time and location of lightning discharge in and around the Observatory. The data would be used to search for correlations between lightning and cosmic ray events. Finally E. Ruigrok et al. describe a seismic sensor array to study wave propagation from distant earthquakes. They contemplate the interesting possibility of using this array to help understanding the sensitivity of the Auger Surface Detector to seismic events.

On a personal note I would say how exciting I found the meeting with many of the topics catching my imagination and it made me wonder what science I would embark on if I had my career over again. I hope that readers of this Focus Point will share some of my excitement.

The meeting would not have been possible without the support of the EU through the Framework 6 programme, the hard organisational work of Gavin Phillips (University of Leeds), the advice on speakers from Mike Pilling and Stephen Mobbs (University of Leeds) and from Jasper Kirkby (CERN). I am grateful to all for their help and support. Antonio Bueno had the idea of a Focus Point and jointly shared the role of editor with Lawrence Wiencke. To both of these Auger colleagues I am very indebted. 